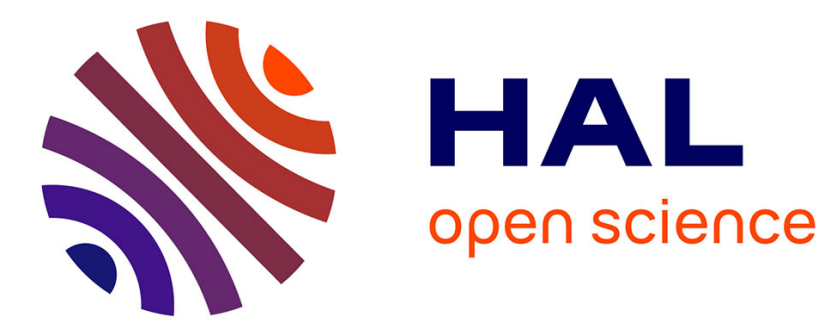

\title{
A new denoising model for multi-frame super-resolution image reconstruction
}

Idriss El Mourabit, Mohammed El Rhabi, Abdelilah Hakim, Amine Laghrib, Eric Moreau

\section{- To cite this version:}

Idriss El Mourabit, Mohammed El Rhabi, Abdelilah Hakim, Amine Laghrib, Eric Moreau. A new denoising model for multi-frame super-resolution image reconstruction. Signal Processing, 2017, 132, pp.51 - 65. 10.1016/j.sigpro.2016.09.014 . hal-01811748

\section{HAL Id: hal-01811748 \\ https: / hal-enpc.archives-ouvertes.fr/hal-01811748}

Submitted on 11 Jun 2018

HAL is a multi-disciplinary open access archive for the deposit and dissemination of scientific research documents, whether they are published or not. The documents may come from teaching and research institutions in France or abroad, or from public or private research centers.
L'archive ouverte pluridisciplinaire $\mathbf{H A L}$, est destinée au dépôt et à la diffusion de documents scientifiques de niveau recherche, publiés ou non, émanant des établissements d'enseignement et de recherche français ou étrangers, des laboratoires publics ou privés. 


\title{
A new denoising model for multi-frame super-resolution image reconstruction
}

\author{
Idriss El Mourabit ${ }^{a}$, Mohammed El Rhabi ${ }^{b}$, Abdelilah Hakim ${ }^{a}$, Amine \\ $\operatorname{Laghrib}^{a} *$ Eric Moreau ${ }^{c}$ \\ ${ }^{a}$ University Cadi Ayyad, Faculty of Science and Technology of Marrakech, Department \\ of Mathematics and Informatics, Marrakech, Morocco. \\ ${ }^{b}$ Applied Mathematics and Computer Science department, Ecole des Ponts ParisTech \\ (ENPC), Paris, France. \\ ${ }^{c}$ CNRS, LSIS, UMR 7296, Université de Toulon, 83957 La Garde, France. \\ *Correspondig author : laghrib.amine@gmail.com
}

\begin{abstract}
Multi-frame image super-resolution (SR) aims to combine the sub-pixel information from a sequence of low-resolution (LR) images to build a highresolution (HR) one. SR techniques usually suffers from annoying restoration artifacts such as noise, jagged edges, and staircasing effect. In this paper, we aim to increase the performance of SR reconstitution under a variational framework using adaptive diffusion-based regularization term. We propose a new tensor based diffusion regularization that takes the benefit from the diffusion model of Perona-Malik in the flat regions and use a nonlinear tensor derived from the diffusion process of weickert filter near boundaries. Thus, the proposed SR approach can preserve important image features (sharp edges and corners) much better while avoiding artifacts. The synthetic and real experimental results show the effectiveness of the proposed regularisation compared to other methods in both quantitatively and visually.
\end{abstract}

Keywords: Super-resolution, Multi-frame, Image restoration, Variational regularization, Tensor diffusion.

\section{Introduction}

Currently, image multi-frame super-resolution reconstruction $[1,2,3,4,5]$ is one of the relevant inverse problems research in image processing. The aim of this technique is to reconstruct a high-resolution (HR) image from a set 
of low-resolution (LR) ones that are noisy, blurred, deformed and downsampled $[6,7]$. Multi-frame SR techniques is used in many applications and various fields. For instance, to achieve high recognition rates of the quality assurance in industries [8,9], agricultural surveillance [10], environmental issues [11], security and video surveillance $[12,13]$ and also cultural heritage $[14,15]$, resolution enhancement is therefore necessary. Moreover, to address the hardware limitations and the very expensive price that sophistication of hardware components requires, the SR technique is preferable, namely in areas like medical diagnostics [16] and satellite imaging [17] such as remote sensing and military surveillance, ... etc.

Multi-frame super-resolution is achieved in three basic steps [18]: first, the motions between each two frames of the captured image sequence are computed which constitutes the registration step ; then, an alignment of this frames onto an HR grid is performed using the motion vectors; and, eventually, a denoising and deblurring step is required to reduce the noise, blur and misregistration errors resulting from the registration step. After the first work proposed in [19], where the authors considered a frequency domain approach, several approaches have been proposed and studied to improve the multi-frame SR problem [20,21, 22, 23, 24]. Earlier works on SR algorithms are based on regularization method due to its ill-posed nature which mainly contains the likelihood and prior function $[25,26]$. The likelihood function measures the difference between the LR images and the obtained HR one, while the image prior function, impose some prior knowledge on the desired HR image. Some of the widely-used prior functions was the Total Variation (TV) regularization as in [27] which avoid the edge-blurring effect caused by the Tikhonov regularization [22]. Besides, some minima estimators (M-estimators) based on statistics knowledge have been explored in multiframe super-resolution algorithm [28]. Namely, the M-estimation method in the context of regularization framework, such as the use of Huber function in the fidelity term [29]. More robust estimators was introduced without regularisation using the Lorentzian error norm [30] and Gaussian error norm [31]. Other robust M-estimation combine with a robust estimators and the Lorentzian error norm in both the fidelity and regularization terms $[32,33]$. The main drawbacks of these methods is the non-convexity of the minimizing function even if the robustness against outliers is significant.

One of the successful and simplest choice of the prior function in the SR problem was the Bilateral Total Variation (BTV) [34], which the HR image is obtained by replacing every pixel with a weighted average of its 
neighborhood. Although the BTV regularization preserves edges typically in non-smooth regions, the use of the Lebesgue $\left(L^{1}\right)$ norm in the regularization term produce artificial edges in the flat surfaces. To overcome the weakness of the BTV term Zeng and Yang proposed the adaptive Bilateral Edge-Preserving (BEP) [35] norm, which stop the diffusion process of the BTV term in smooth areas of the image. In the same principle, another adaptive combined of the TV and BTV norms was introduced to avoid the straicasing effect, which increase the performance of the restoration step of the SR algorithm [36]. Recently, an adaptive diffusion-based regularizer was treated to preserve important image features [37]. Even if this approach avoids undesirable artifacts while suppressing noise, it suffers from the blurring effect.

The main goal of this paper consists of increasing the robustness of the super-resolution techniques over the methods discussed above with respect to the misregistration errors, blurring effect and noise. To avoid the errors arising from the restoration step, we use a nonlinear diffusion-based regularizer derived from the Weickert filter [38, 39, 40]. Since the Weickert process destroys edges and generates curved structure under intense noise conditions, improvements of this filter are desired. In the context of super-resolution, we propose a more robust filter against noise and blur, which takes into account the coherence-enhancing property. This approach takes into consideration the best of Perona-Malik processe in flat regions [41], and the benefit of the Weickert filter effect near sharp edges. The proposed regularizer has proved its efficiency in avoiding the staircasing effect and blur while reducing noise, which can preserve image features much better compared with the existing diffusion-based regularizers.

The outline of the paper is the following one. In Section 2, we present the general multi- frame super-resolution problem. Then, we set the derived regularized criterion. After, we introduce the variational problem and we prove the existence of a solution of the proposed equation. In section 3 , we present some experimental results, while we compare our approach with some available methods. We finally end the paper by a conclusion.

\section{Muti-frame SR problem formulation}

The observed images of a real scene usually are in low resolution. This is due to some degradation operators. In practice, the acquired images are decimated, corrupted by noise and suffered from blurring [18]. We assume that 
all low resolution images are taken under the same environmental conditions using the same sensor. The relationship between an ideal HR image $X$ (represented by a vector of size $\left[r^{2} N^{2} \times 1\right]$, where $r$ is the resolution enhancement factor) and the corresponding LR ones $Y_{k}$ of size $N \times N$ (represented by a vector of size $\left.\left[N^{2} \times 1\right]\right)$, is described by the following model

$$
Y_{k}=W F_{k} H X+V_{k} \quad \forall k=1,2, \ldots, n,
$$

where

$n$ : the number of LR frames.

$H$ : the blurring operator of size $\left[r^{2} N^{2} \times r^{2} N^{2}\right]$.

$W$ : represents the decimation matrix of size $\left[N^{2} \times r^{2} N^{2}\right]$.

$F_{k}$ : is a geometric warp matrix of size $\left[r^{2} N^{2} \times r^{2} N^{2}\right]$, representing a nonparametric transformation that differs in all frames.

$V_{k}$ : is a vector of size $\left[N^{2} \times 1\right]$ which represents the additive noise for each image.

Given a LR sequence $Y_{k}, k=1, \ldots, n$, the aim of SR consists of reconstructing the original image $X$. Since the super-resolution problem is ill-posed (the solution is not unique), the reconstitution is very unstable. This can be fixed by requiring some prior knowledge about the image $X$ in a Bayesian framework. Via the the maximum a posteriori (MAP) estimator, the estimation of HR image is given through the following minimization problem

$$
\begin{aligned}
\widehat{X} & =\arg \max _{X}\left\{p\left(X / Y_{k}\right)\right\} \\
& =\arg \max _{X}\left\{p\left(Y_{k} / X\right) \cdot p(X)\right\} \\
& =\arg \min _{X}\left\{-\log \left(p\left(Y_{k} / X\right)\right)-\log (p(X))\right\}
\end{aligned}
$$

where $p\left(Y_{k} / X\right)$ represents the fidelity term (i.e. the relationship between the observed images and the HR one), while $p(X)$ describes the prior knowledge imposed on the high-resolution image $X$. Substituting these two terms by their expressions, we define the general super-resolution model as follows :

$$
\widehat{X}=\underset{\mathbf{x}}{\arg \min }\left\{\sum_{1}^{n}\left\|W F_{k} H X-Y_{k}\right\|_{2}+\delta R(X)\right\},
$$

where $R(X)$ is a regularisation term representing a prior knowledge on $X$. There is a great number of proposed regularisations in the multi-frame superresolution context $[36,35,34,26]$. The main principle of these approaches 
is to provide a global minimum to the encountered minimization problem. With the same aim, recently, Maiseli et al. [37] proposed a new regularizing potential defined by

$$
R(X)=\int_{\Omega} \frac{\frac{|\nabla X|}{\beta}\left(2+\frac{|\nabla X|}{\beta}\right)}{1+\left(\frac{|\nabla X|}{\beta}\right)^{2}} \mathrm{~d} \Omega,
$$

where $\beta$ is a shape-defining tuning constant. The purpose of this choice is to combine the edge preserving effect of the total variation [42] with the Perona-Malik diffusion behavior [41] and the effect smoothing of backward diffusion anisotropic [43]. Which can preserve sharpen edges and details. The corresponding evolution equation of the Euler- Lagrange form of (3) using the regularisation term in (4) is

$$
\frac{\partial X}{\partial t}=\frac{1}{n} \sum_{k=1}^{n} W^{\top} F_{k}^{\top} H^{\top}\left(W F_{k} H X-Y_{k}\right)+\operatorname{div}\left(\frac{2+\frac{|\nabla X|}{\beta}}{1+\left(\frac{|\nabla X|}{\beta}\right)^{2}} \nabla X\right) .
$$

Even if this equation preserves feature of the image, it suffers from the blurring effect in the homogeneous regions. To address this problem, we propose a new equation that performs the coherence- enhancing property and avoids blur. This approach takes in consideration the best of Perona-Malik diffusion process in flat regions [41], and the benefit of Weickert filter effect [38] near sharp edges and corners. The proposed equation with Neumann boundary conditions is given as follows

$$
\left\{\begin{array}{l}
\left.\frac{\partial X}{\partial t}(t, x)-\operatorname{div}\left(D\left(J_{\rho}\left(\nabla X_{\sigma}\right)\right) \nabla X\right)-\frac{1}{m} \sum_{i=1}^{m}\left(W F_{k} H\right)^{\top}\left(W F_{k} H X-Y_{k}\right)=0 \text { on }\right] 0, T[\times \Omega, \\
\left.\left\langle D\left(J_{\rho}\left(\nabla X_{\sigma}\right)\right) \nabla X, n\right\rangle=0 \text { on }\right] 0, T[\times \partial \Omega, \\
X(0, x)=X_{0}(x),
\end{array}\right.
$$

where $D$ is an anisotropic diffusion tensor and $J_{\rho}$ is the structure tensor defined by

$$
J_{\rho}\left(\nabla X_{\sigma}\right)=K_{\rho} *\left(\nabla X_{\sigma} \otimes \nabla X_{\sigma}\right)=K_{\rho} *\left(\nabla K_{\sigma} * X \nabla K_{\sigma} * X^{\top}\right) .
$$

With $K_{\rho}$ and $K_{\sigma}$ represents a two Gaussian convolution kernels such as $K_{\tau}(x)=\frac{1}{2 \pi \tau^{2}} \exp \left(-\frac{|x|^{2}}{2 \tau^{2}}\right)$. The function $D$ is chosen in order to preserve 
edges and corners as much as possible. This function is computed using the eigenvalues and the eigenvectors of the structure tensor $J_{\rho}$ as follows

$$
D:=f_{+}\left(\lambda_{+}, \lambda_{-}\right) \theta_{+} \theta_{+}^{\top}+f_{-}\left(\lambda_{+}, \lambda_{-}\right) \theta_{-} \theta_{-}^{\top},
$$

where $\lambda_{+/-}$and $\theta_{+/-}$are respectively the eigenvalues and the eigenvectors of the tensor structure $J_{\rho}$, the eigenvalues $\lambda_{+/-}$are calculated as

$$
\lambda_{+/-}=\frac{1}{2}\left(\operatorname{trace}\left(J_{\rho}\right) \pm \sqrt{\operatorname{trace}^{2}\left(J_{\rho}\right)-4 \operatorname{det}\left(J_{\rho}\right)}\right) .
$$

This choice is justified using the geometric characteristics of the restored image, for more details see [44]. Indeed, if $\lambda_{+/-} \approx 0$, the smoothing within homogeneous areas is isotropic, while if $\lambda_{+} \gg \lambda_{-} \approx 0$, the smoothing process is anisotropic and directed along the straight edges. However, if $\lambda_{+} \gg \lambda_{-} \gg 0$, the smoothing takes into account corners. Also, the functions $f_{+}\left(\lambda_{+}, \lambda_{-}\right)$and $f_{-}\left(\lambda_{+}, \lambda_{-}\right)$are chosen carefully in order to satisfy the following smoothing constraints:

1. Isotropic: in the homogeneous areas the diffusion process should be isotropic.

2. Anisotropic: near of sharp edges, the diffusion process should be anisotropic.

Many choices have been proposed for the functions $f_{+/-}$, namely, the functions associated to the Perona Malik process are defined as

$$
f_{+/-}\left(\lambda_{+}, \lambda_{-}\right)=\exp \left(-\frac{\left(\lambda_{+}+\lambda_{-}\right)^{2}}{k}\right) .
$$

Using this function, the diffusivity matrix $D$ has no specific orientation and it stops diffusion near edges and corners. To outperform the defects of the Perona Malik model, the model of Weickert [39] has been proposed choosing $f_{+}$and $f_{-}$as follows

$$
\left\{\begin{array}{l}
f_{+}\left(\lambda_{+}, \lambda_{-}\right)= \begin{cases}\alpha+(1-\alpha) \exp \left(-\frac{k}{\left(\lambda_{+}-\lambda_{-}\right)^{2}}\right) & \text { if } \quad \lambda_{+} \neq \lambda_{-}, \\
\alpha & \text { else } \quad(\alpha=0.001),\end{cases} \\
f_{-}\left(\lambda_{+}, \lambda_{-}\right)=\alpha
\end{array}\right.
$$

The Weickert coherence enhancing diffusion approach [44] leads to good results in the case of anisotropic process, particularly, the diffusion is oriented 
in one direction. However, the Weickert model destroys corners and singularities of the restored image. To overcome this issue, we propose new functions which leads to skipping the behaviour of Weickert coefficients and allows to diffuse in two directions $\theta_{+}$and $\theta_{-}$. These functions are presented as follows

$$
\left\{\begin{array}{l}
f_{+}\left(\lambda_{+}, \lambda_{-}\right)=\exp \left(-\frac{\lambda_{+}}{k_{1}}\right) \\
f_{-}\left(\lambda_{+}, \lambda_{-}\right)=\exp \left(-\frac{\lambda_{-}}{k_{2}}\right)\left(1-\exp \left(-\frac{\lambda_{+}}{k_{1}}\right)\right)
\end{array}\right.
$$

where $k_{1}$ and $k_{2}$ are two thresholds which define the diffusion along the directions $\theta_{+}$and $\theta_{-}$respectively. Since the desired diffusion changes according to the image feature: contour or singularity, the parameters $k_{1}$ and $k_{2}$ are used to define the sensitivity threshold that determine the variation in a given image. With this choice, the PDE in (6) allows to reduce noise in uniform zones, smooth edges and preserve singularities correctly.

Since the restoration step is an ill-posed problem [45], we have to check the existence and uniqueness of the proposed PDE (6). We first recalls some interesting assumptions:

1. There exists $\eta_{k}>0$ such that $\left\langle N_{k} Y, Y\right\rangle \leq \eta_{k}\|Y\|^{2}$, with

$$
N_{k}=\left(W F_{k} H\right)^{\top} W F_{k} H, \quad \forall k=1, \ldots n .
$$

2. The diffusion tensor $D$ is infinitely continuous, i.e $D \in \mathcal{C}^{\infty}\left(\mathbb{R}^{2 \times 2}, \mathbb{R}^{2 \times 2}\right)$.

3. $D$ is a symmetric and positive-definite matrix.

In the following theorem, we prove the existence and uniqueness of solution to $(6)$.

Theorem 2.1. Let $X_{0} \in \mathbf{L}^{\infty}(\Omega), \rho \geq 0$ and $\sigma, T>0$. Under the assumptions above, there exists a unique function $X(t, x) \in C\left((0, T) ; \mathbf{L}^{2}(\Omega)\right) \cap$ $\mathbf{L}^{2}\left((0, T) ; \mathbf{H}^{1}(\Omega)\right)$ satisfying the equation (6). Moreover, the solution depends continuously on $X_{0}$ with respect to $\|\cdot\|_{\mathbf{L}^{2}(\Omega)}$, and $X \in C((0, T) \times \bar{\Omega})$.

proof 1. See Appendix.

Since we have demonstrated the existence and uniqueness of the solution to the proposed equation, the convergence of the proposed scheme is assured. Indeed, we solve the problem (6) using an explicit finite difference scheme [44]. We will not detail this part, since it is similar to previous work [36, 37], where an appropriate scheme is used to discretize the proposed PDE. This 
scheme is computationally fast, efficient, and mathematically well posed as demonstrated in $[37,44]$. To compute the warping matrix $F_{k}$ (to align the input images), we used a sub-pixel accurate optical flow method [46]. In this approach, a variational minimization problem is used with a Huber-Norm regularization and an $L^{1}$ based data in the optical flow constraint model.

To observe the robustness of the proposed regularizer in (6) with respect to noise, we consider a simple denoising problem. For that, we take $m=1$ and $W F_{k} H=I$ in (6), we select the image in Fig. 1 (a) and added to it a zero mean Gaussian noise with standard deviation $\sigma_{\text {noise }}=25$, then, we applied the nonlinear diffusion regularizer of Perona-Malik (PM) [41], Weickert et al. [38], BTV [34] and ours, to generate the results in Fig. 1. Concerning the parameter selection in equation (10), the two threshold $k_{1}$ and $k_{2}$ are chosen carefully with respect to the best obtained PSNR value as shown in the Fig. 2. We use the same thing to select the parameter $\sigma$ (see Fig. 2). For the parameter $\rho$, weickert demonstrated in [38] that in general $\rho=3 \sigma$. We recall that the choice of these parameters depends strictly on the nature of the image, the level of noise and loss continuity of lines. We use the same techniques to determine $k_{1}, k_{2}$ and $\sigma$, after, in the simulation experiments within the SR framework. Figs. 3, 4 and 5 show the evolution of the PSNR up to 120 iterations of the reconstruction results (with $\sigma_{\text {noise }}=25, \sigma_{\text {noise }}=30$ and $\sigma_{\text {noise }}=35$ respectively), using different regularizers. If we look at the evolution of the PSNR associated with our approach, we can deduce that the curve is not too much affected by increasing noise levels compared with the other methods. Which once again demonstrates the effectiveness of the proposed PDE.

\section{Numerical Result}

In this section various simulated and also real results for assessing the performance of the proposed multi-frame SR method will be presented. Comparison with some available and competitive multi-frame SR method is carried out. Namely, TV [47], BTV [34], TV+BTV [36], and nonlinear diffusion regularizer [37]. The proposed super-resolution method is tested on a large benchmark image, we present only four of them. In the first simulated experiment, we degraded each of the original HR images of the Castle, EIAcen, Champignon, and Penguin to generate the corresponding sequence of twenty synthetic LR images. This sequence is obtained from the original images such as each frame is blurred by a Gaussian low-pass filter with a $3 \times 3$ and 


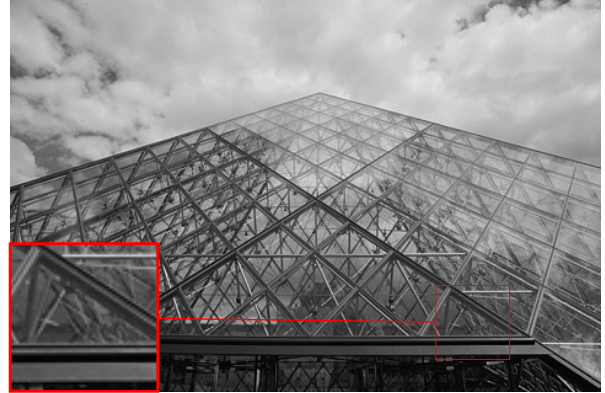

(a) Original

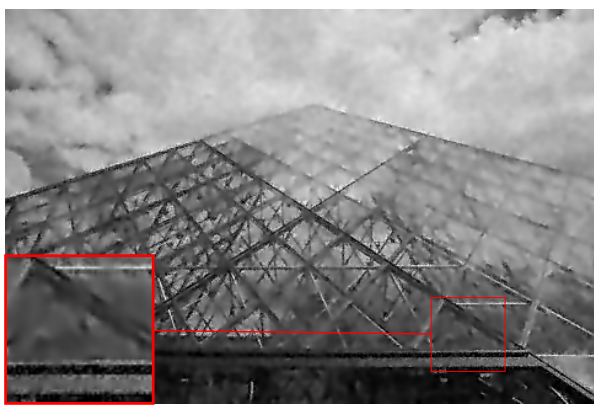

(c) PM [41]

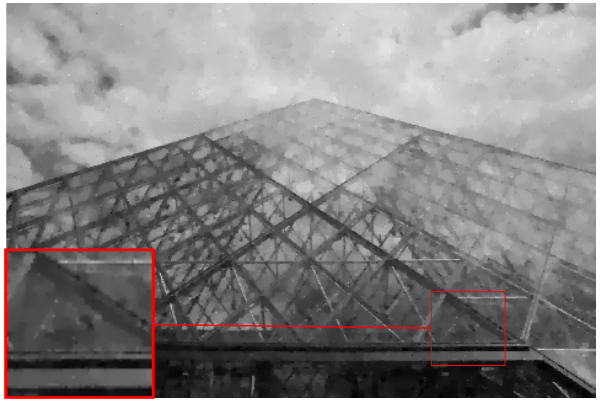

(e) BTV [34]

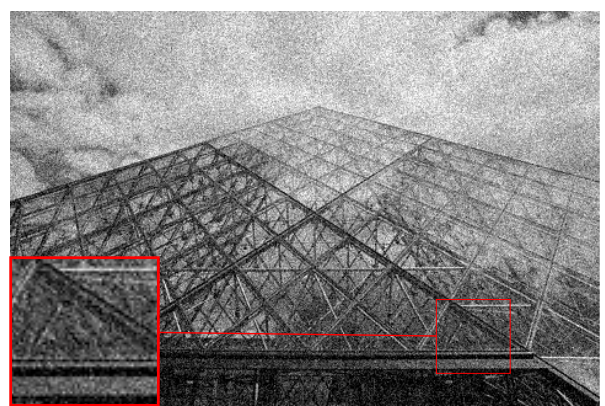

(b) Noisy

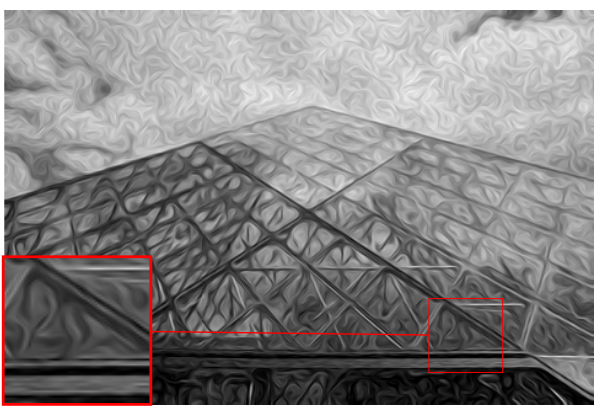

(d) Weickert et al. [38]

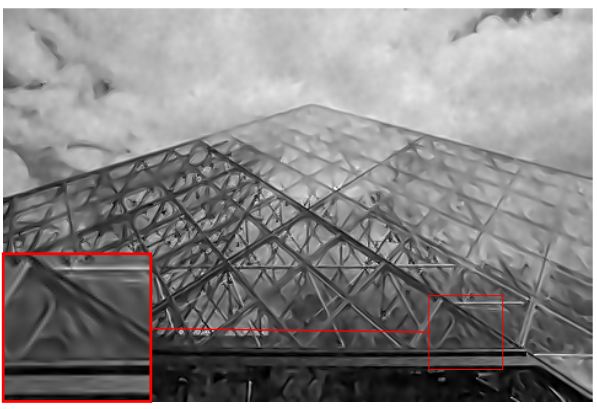

(f) Our method

Figure 1: The denoising process using different regularizers compared with our approach. 


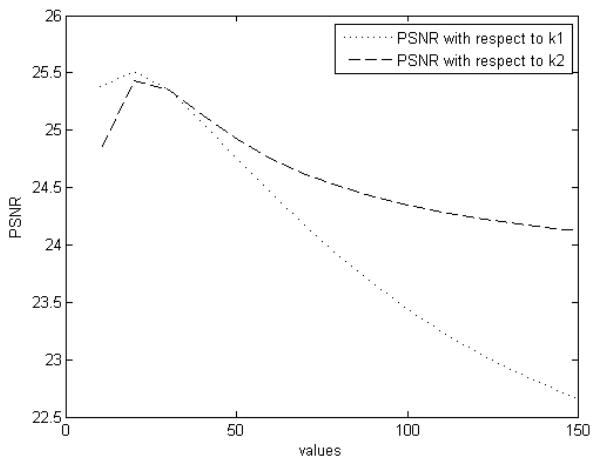

(a) The parameters $k_{1}$ and $k_{2}$

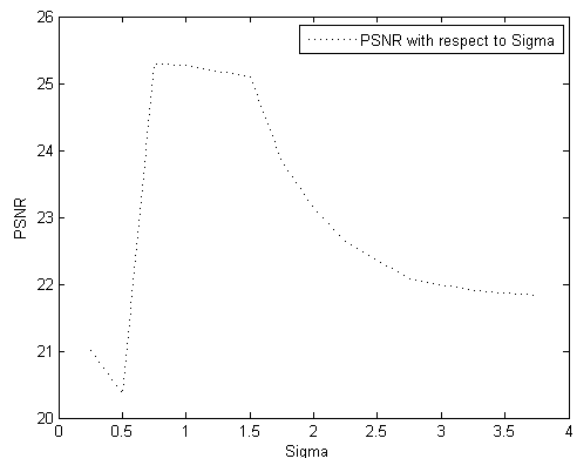

(b) The parameter $\sigma$

Figure 2: The variation of the PSNR value with respect to the parameters $k_{1}$ (fixing $\left.k_{2}=30\right), k_{2}$ (fixing $\left.k_{1}=30\right)$ and $\sigma$.

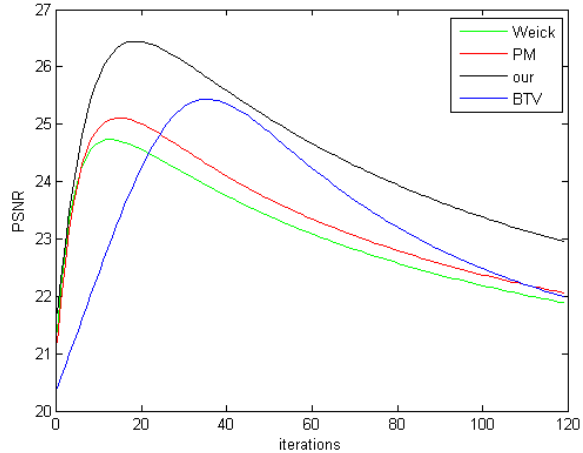

(a) The PSNR value

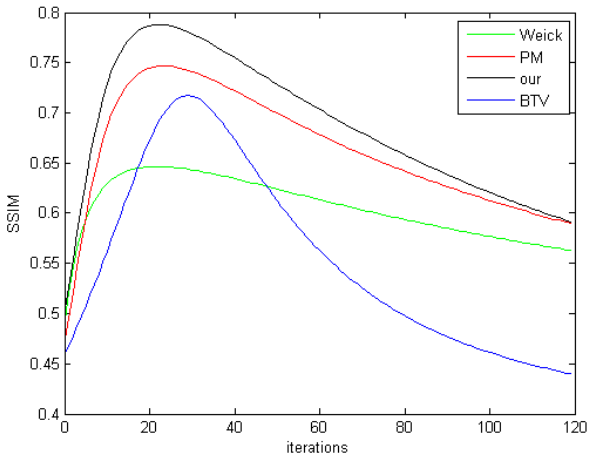

(b) The SSIM value

Figure 3: The variation of the PSNR and SSIM values (for the obtained images in figure 1) with respect to number of iterations for different regualizers. 


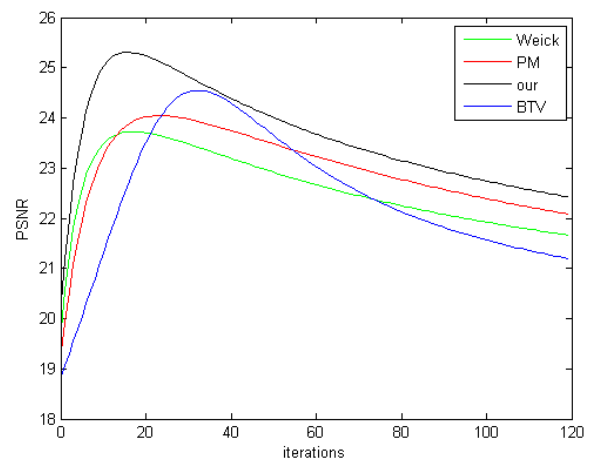

(a) The PSNR value

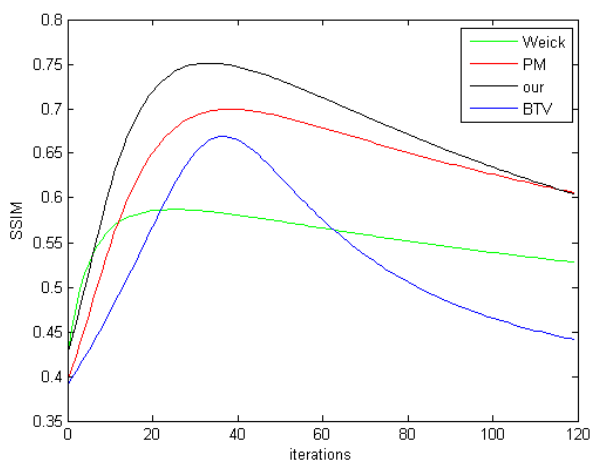

(b) The SSIM value

Figure 4: The variation of the PSNR and SSIM values (with $\sigma_{\text {noise }}=30$ ) with respect to number of iterations for different regualizers.

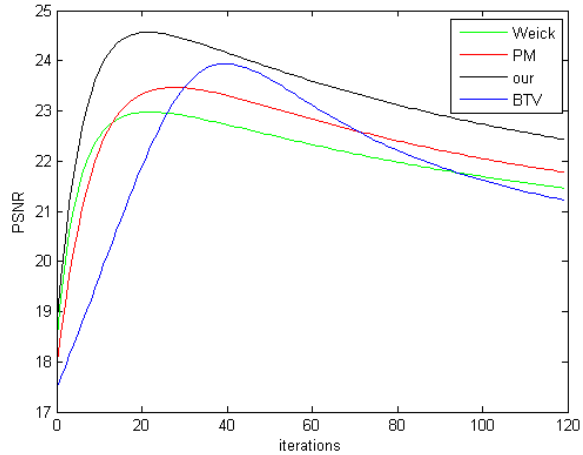

(a) The PSNR value

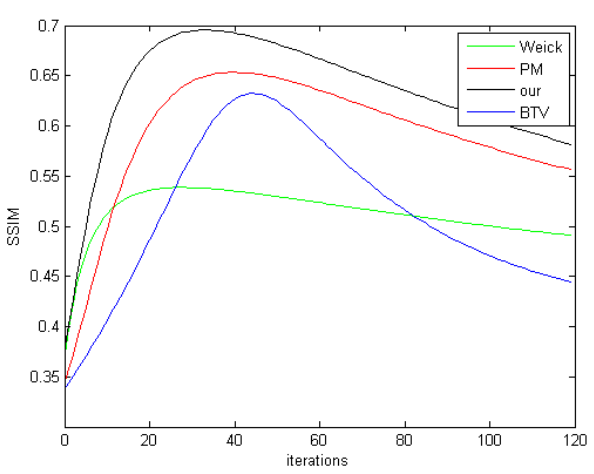

(b) The SSIM value

Figure 5: The variation of the PSNR and SSIM values (with $\sigma_{\text {noise }}=35$ ) with respect to number of iterations for different regualizers. 
standard deviation of 1.5. Then, the blurred frames are down-sampled vertically and horizontally by a factor of $r=4$ and Gaussian noise was finally added, to down-sampled frames, with $\sigma_{\text {noise }}=20$. Afterwards, we applied various multi-frame SR method chosen above, and the proposed method to reconstruct the respective original images of the degraded ones (represented in Figs. 6 to 9). To test and compare the performance of the proposed method with the others, a quantitative evaluation is needed, we have used two metrics: peak-signal-to-noise ratio (PSNR) [48], and mean structure similarity (SSIM) [49]. The PSNR measures signal strength relative to noise in the image and is defined by

$$
P S N R=10 \log _{10}\left(\frac{255^{2}}{M S E}\right)
$$

where the $M S E$ is the mean squared error defined by

$$
M S E=\frac{1}{M N} \sum_{i=1}^{M} \Sigma_{j=1}^{N}(Y(i, j)-X(i, j))^{2} .
$$

The $S S I M$ is calculated on multiple windows of given image, i.e. the measurement between two windows $x$ and $y$ of size $N \times N$ is defined by

$$
\operatorname{SSIM}(x, y)=\frac{\left(2 \mu_{x} \mu_{y}+c_{1}\right)\left(2 \sigma_{x} \sigma_{y}+c_{2}\right)\left(2 \operatorname{cov}_{x y}+c_{3}\right)}{\left(\mu_{x}^{2}+\mu_{y}^{2}+c_{1}\right)\left(\sigma_{x}^{2}+\sigma_{y}^{2}+c_{2}\right)\left(\sigma_{x} \sigma_{y}+c_{3}\right)},
$$

where the variables, respectively, defined for $x$ and $y$ as follows: $\mu_{x}$ and $\mu_{y}$, mean; $\sigma_{x}^{2}$ and $\sigma_{y}^{2}$, variance; $\operatorname{cov}_{x y}$, covariance; $c_{1}=\left(k_{1} L\right)^{2}, c_{2}=\left(k_{2} L\right)^{2}$ are two stabilizing constants; and $L$ the dynamics of the pixel values, 255 for 8-bit encoded image. This metric gives an indication on the quality of the image based on the known characteristics of human visual system.

The obtained results in Figs. (6)-(9) demonstrate that, visually, the proposed method outperforms the others. Moreover, the quantitative results presented in the tables 1 and 2 confirm that the proposed model is usually with the higher PSNR and SSIM values. Note that we select the optimal parameters according to the best PSNR value in all the experiments for the other methods. Table 1 shows that our method is always with the best PSNR values in most cases, which affirm its robustness against noise and blur reduction. Also, the obtained SSIM values in Table 2 confirms the high quality of the obtained image in terms of details image preserving. Characteristically, the execution of the main implementation on MATLAB R2014 requires on 
the average about $7 \sim 14$ second on a i7 $3.4 \mathrm{GHz}$ Quad-Core computer for $256 \times 256$ images. Effectively, the time becomes quite large for the color and large-size images. Furthermore, we resume the necessary execution time to increase the resolution by a factor of $r=4$ of the proposed algorithm compared with the others in Table 3.

To measure the robustness of the proposed approach against noise, we add another test which the Gaussian noise is added with $\sigma_{\text {noise }}=50$ to all 100 generated LR Fish and Barbara images. Fig. 10 shows the obtained result (for the Fish image) using the proposed algorithm (for different choices of $k_{1}$ and $k_{2}$ values) compared with other approaches. By a visual evaluation, we can see the robustness of the proposed method in reducing noise with the capability of a better edge recovery compared to other methods. Moreover, as is shown in Table 4, quantitative results confirm that our proposed approach has, usually, the best performance in reconstruction in terms of the PSNR values. In addition, we can see that our approach is robust against noise compared with the others. Since, for example, we obtain the best PSNR value in the image of Barbara for $\sigma_{\text {noise }}=50$, while for $\sigma_{\text {noise }}=10$, Maiseli et al. [37] method was the best. Despite the promising results, the proposed method suffers from the weakness that it does not preserve as much the texture. A deep research is thus needed to address this problem.

The final experiment is for a real video sequence called "Text" downloaded from the website ${ }^{1}$, which are a challenging example, since it contain a high level of outliers. We use the eight first images of the "Text" video as a LR sequence of size $49 \times 57$, and the size of the reconstructed images is $98 \times 114$. The reconstructed image by different methods are presented in the Fig. 11. Visually, the obtained results show a significant improvement in our method over the others.

Finally, we can summarise that both simulated and real results show that the new approach produces a sharper image with less blur compared with the other methods.

\section{Conclusion}

In this paper, we have presented an new multi-frame super-resolution approach that robustly restores image features from a much noised and blurred

\footnotetext{
${ }^{1}$ https://users.soe.ucsc.edu/ milanfar/software/sr-datasets.html
} 


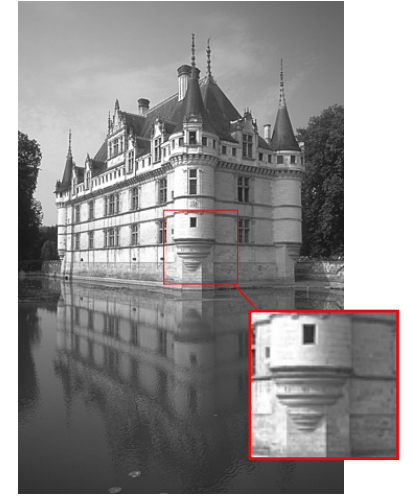

(a) HR image

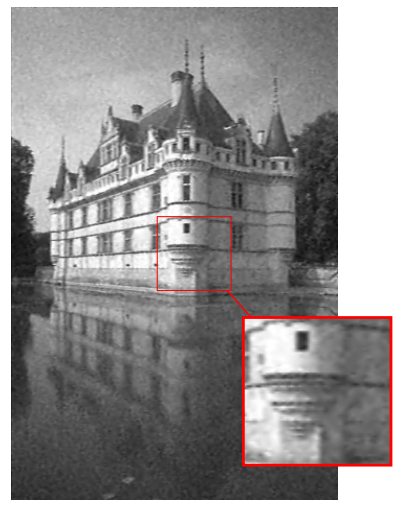

(d) Markina et al. [47]

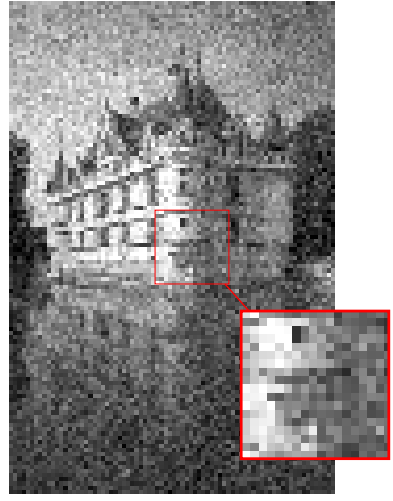

(b) One LR image

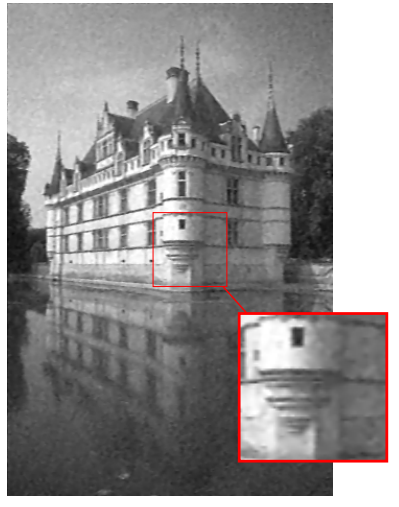

(e) Maiseli et al. [37]

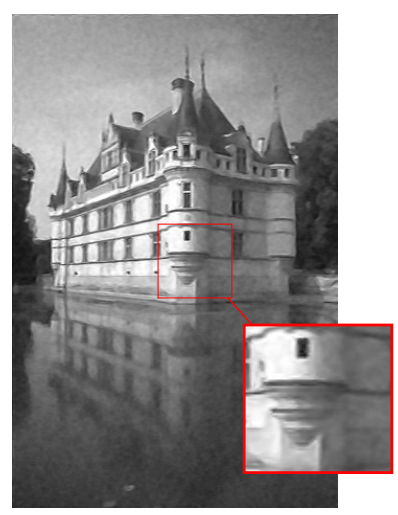

(g) our method

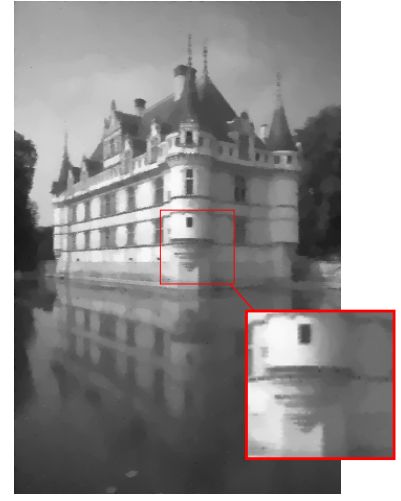

(c) Farsiu et al. [34]

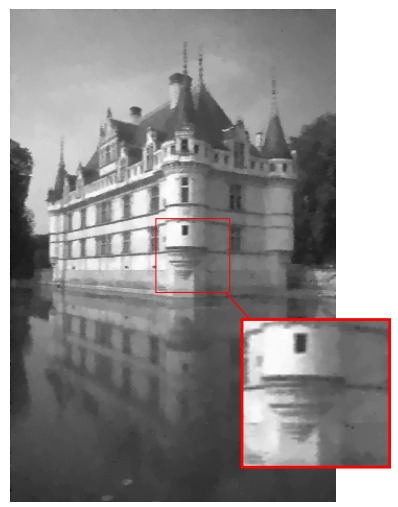

(f) Laghrib et al. [36]

(g)

Figure 6: The results obtained by applying different methods to LR (Castle sequence). 


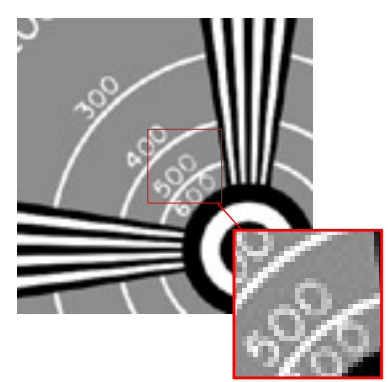

(a) HR image

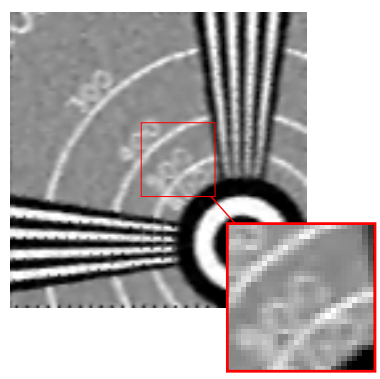

(d) Markina et al. [47]

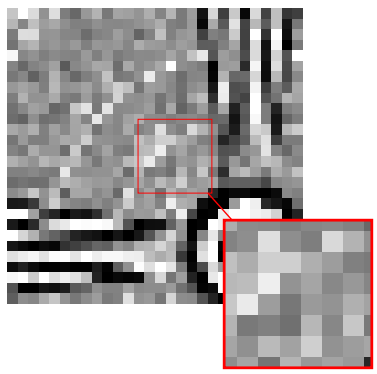

(b) One LR image

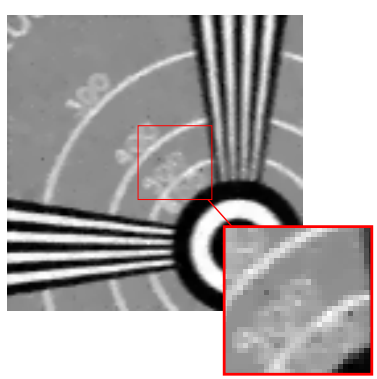

(e) Maiseli et al. [37]

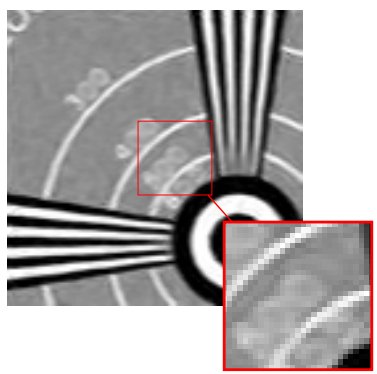

(g) our method

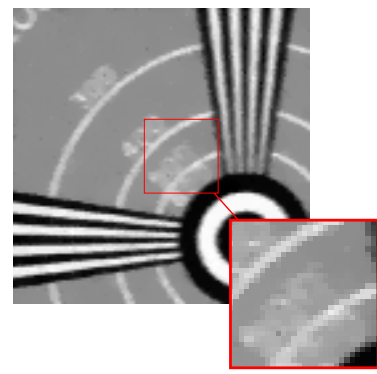

(c) Farsiu et al. [34]

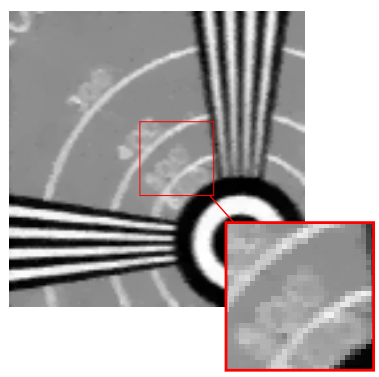

(f) Laghrib et al. [36]

Figure 7: The results obtained by applying different SR methods to LR EIAcen sequence. 


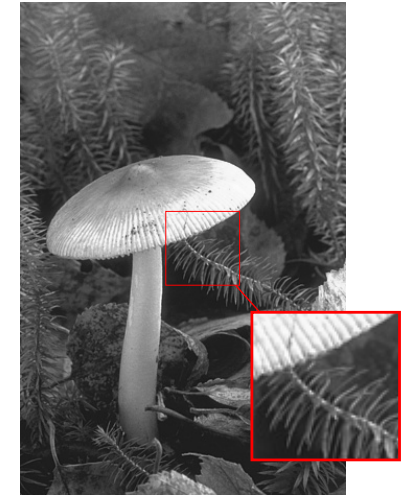

(a) HR image

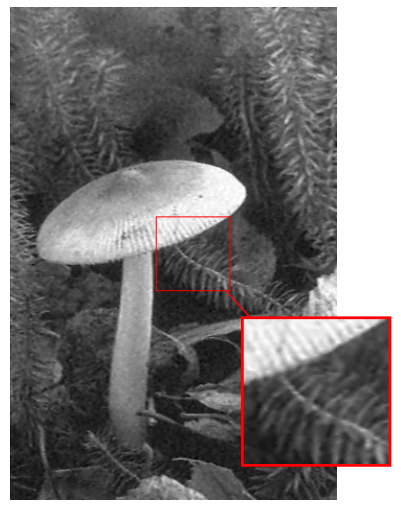

(d) Markina et al. [47]

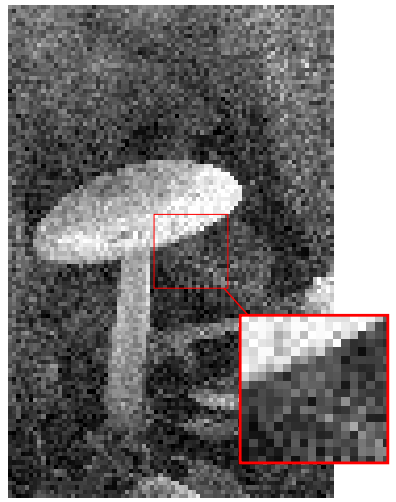

(b) One LR image

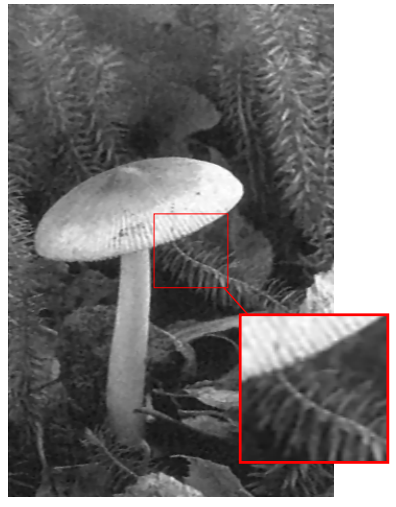

(e) Maiseli et al. [37]

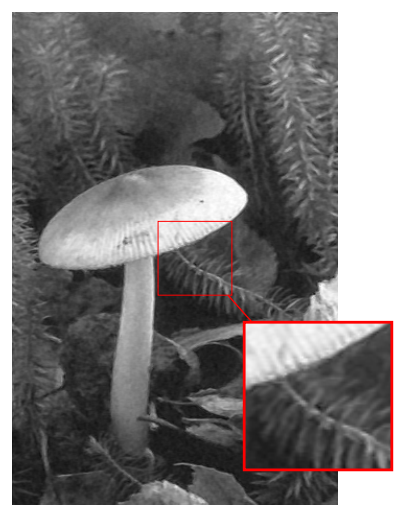

(g) our method

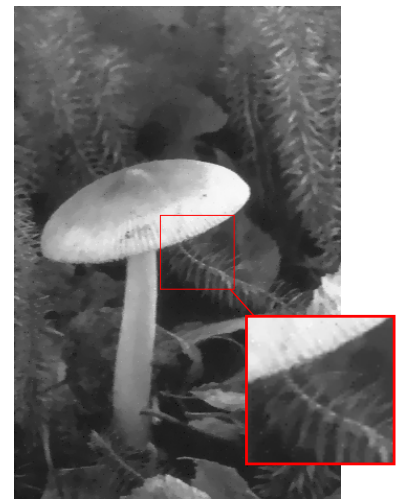

(c) Farsiu et al. [34]

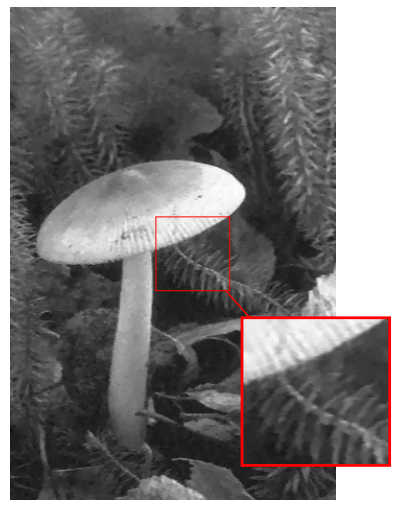

(f) Laghrib et al. [36]

(g) our method

Figure 8: The results obtained by applying different methods to LR (Champignon sequence). 


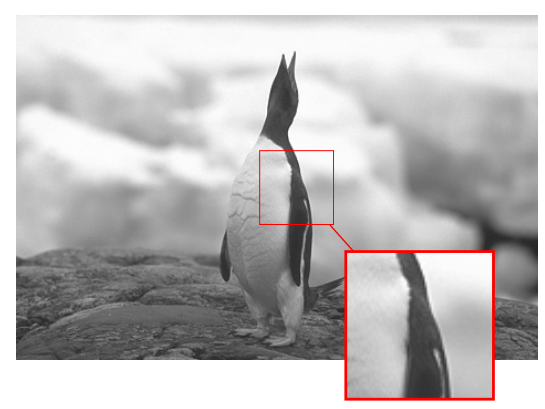

(a) HR image

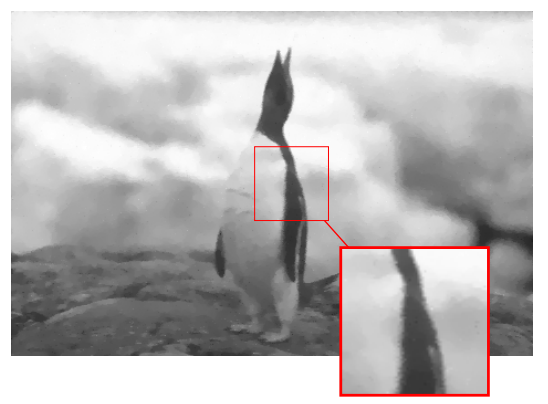

(c) Farsiu et al. [34]

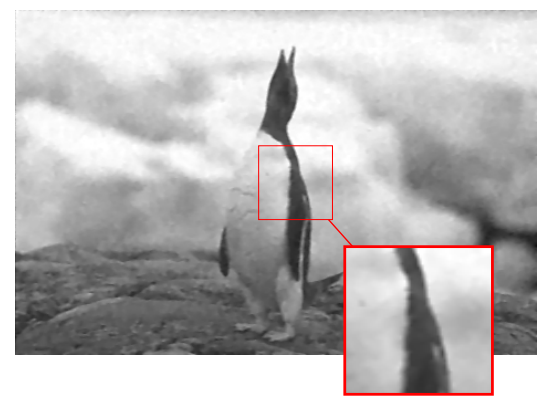

(e) Maiseli et al. [37]

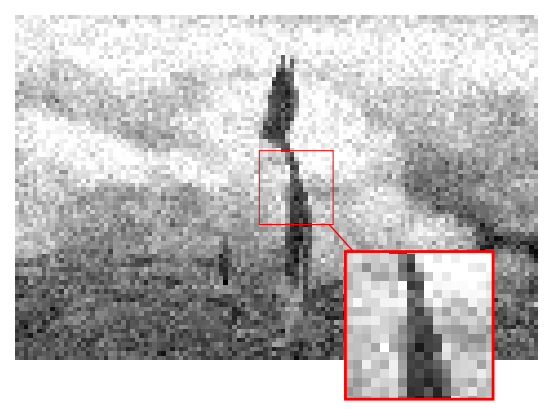

(b) One LR image

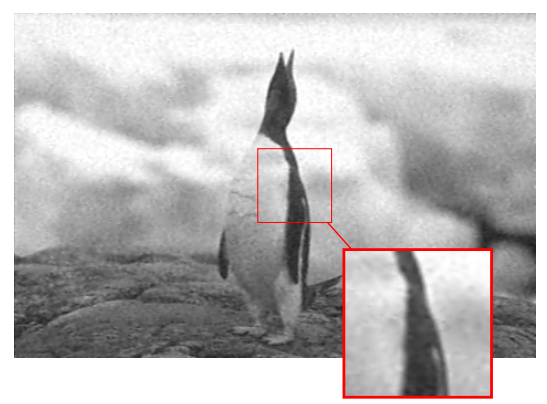

(d) Markina et al. [47]

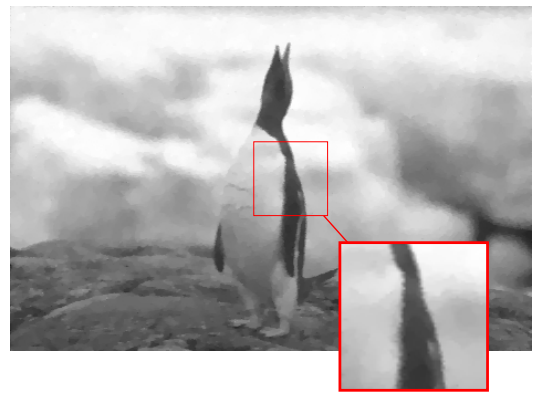

(f) Laghrib et al. [36]

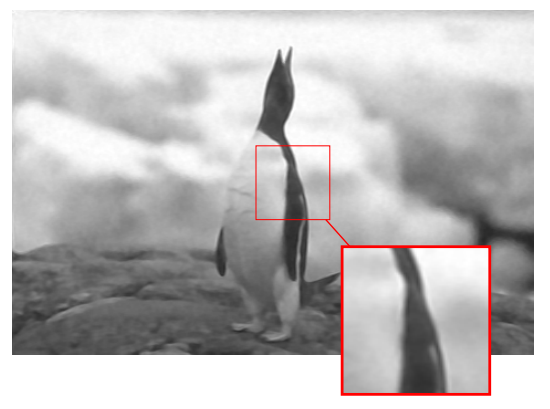

(g) our method

Figure 9: The results obtained by applying different methods to (Penguin sequence). 
Table 1: The PSNR table

\begin{tabular}{cccccc}
\hline \hline \multirow{2}{*}{ Image } & \multicolumn{5}{c}{ PSNR } \\
\cline { 2 - 6 } & Farsiu [34] & Markina et al. [47] & Maiseli et al. [37] & Laghrib et. al [36] & Our Method \\
\hline Castle & 25.70 & 27.02 & 27.13 & 26.56 & $\mathbf{2 7 . 2 8}$ \\
EIAcen & 20.63 & 20.55 & 21.12 & 20.87 & $\mathbf{2 2 . 1 1}$ \\
Champignon & 27.87 & 29.24 & 29.27 & 29.06 & $\mathbf{2 9 . 9 0}$ \\
Penguin & 32.04 & 31.57 & 32.37 & 32.18 & $\mathbf{3 3 . 0 5}$ \\
Lena & 29.08 & 29.15 & 29.84 & 29.11 & $\mathbf{3 0 . 2 2}$ \\
Bird & 33.96 & 33.92 & 34.60 & 34.84 & $\mathbf{3 5 . 0 8}$ \\
Lake & 30.64 & 30.88 & 31.04 & 31.73 & $\mathbf{3 1 . 9 0}$ \\
Baboon & 27.22 & 27.49 & 27.48 & 27.59 & $\mathbf{2 7 . 9 8}$ \\
Peppers & 29.94 & 30.66 & $\mathbf{3 1 . 4 5}$ & 31.18 & 31.06 \\
Goldhill & 29.40 & 29.34 & 30.11 & 29.93 & $\mathbf{3 0 . 3 8}$ \\
\hline Average & 28.64 & 28.98 & 29.44 & 29.30 & $\mathbf{2 9 . 9 9}$ \\
\hline \hline
\end{tabular}

Table 2: The SSIM table

\begin{tabular}{cccccc}
\hline \hline \multirow{2}{*}{ Image } & \multicolumn{5}{c}{ SSIM } \\
\cline { 2 - 5 } & Farsiu [34] & Markina et al. [47] & Maiseli et al. [37] & Laghrib et. al [36] & Our Method \\
\hline Castle & 0.776 & 0.763 & 0.794 & $\mathbf{0 . 8 0 6}$ & 0.803 \\
EIAcen & 0.865 & 0.837 & 0.877 & 0.745 & $\mathbf{0 . 8 8 0}$ \\
Champignon & 0.738 & 0.805 & 0.815 & 0.802 & $\mathbf{0 . 8 3 0}$ \\
Penguin & 0.876 & 0.806 & 0.880 & 0.879 & $\mathbf{0 . 8 8 4}$ \\
Lena & 0.822 & 0.831 & 0.836 & 0.844 & $\mathbf{0 . 8 5 2}$ \\
Bird & 0.864 & 0.852 & 0.871 & 0.873 & $\mathbf{0 . 8 9 2}$ \\
Lake & 0.803 & 0.810 & 0.818 & 0.822 & $\mathbf{0 . 8 2 8}$ \\
Baboon & 0.721 & 0.728 & 0.736 & 0.745 & $\mathbf{0 . 7 5 0}$ \\
Peppers & 0.752 & 0.749 & 0.769 & 0.763 & $\mathbf{0 . 7 7 4}$ \\
Goldhill & 0.830 & 0.821 & 0.865 & 0.869 & $\mathbf{0 . 8 7 8}$ \\
\hline Average & 0.804 & 0.800 & 0.826 & 0.814 & $\mathbf{0 . 8 4 7}$ \\
\hline \hline
\end{tabular}

Table 3: CPU times (in seconds) of different super-resolution methods and the proposed method when the magnification factor is 4 .

\begin{tabular}{cccccc}
\hline \hline \multirow{2}{*}{ Image } & \multicolumn{5}{c}{ CPU Time } \\
\cline { 2 - 6 } & Farsiu [34] & Markina et al. [47] & Maiseli et al. [37] & Laghrib et. al [36] & Our Method \\
\hline Castle & 7.24 & 7.26 & 8.02 & 7.22 & 7.96 \\
EIAcen & 6.22 & 6.64 & 7.62 & 6.34 & 7.61 \\
Champignon & 7.31 & 7.48 & 8.16 & 7.62 & 8.08 \\
Penguin & 7.66 & 7.75 & 8.84 & 7.92 & 8.93 \\
Lena & 9.05 & 9.18 & 10.86 & 9.22 & 10.80 \\
Bird & 8.22 & 8.19 & 9.67 & 8.26 & 9.54 \\
Lake & 8.16 & 8.14 & 10.32 & 8.22 & 10.24 \\
Baboon & 7.87 & 7.96 & 8.66 & 7.88 & 8.60 \\
Peppers & 8.02 & 8.16 & 9.22 & 8.36 & 9.76 \\
Goldhill & 8.12 & 8.59 & 9.98 & 8.41 & 10.02 \\
\hline Average time & 7.78 & 7.93 & 9.13 & 7.94 & 9.15 \\
\hline \hline
\end{tabular}




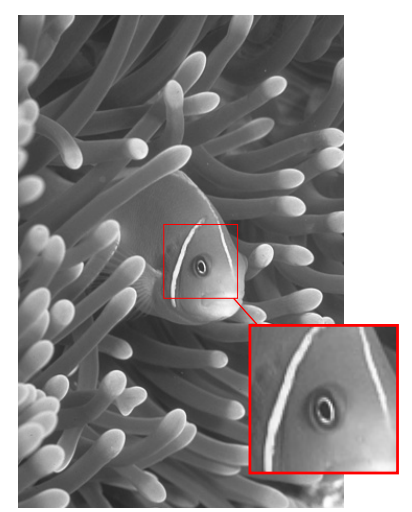

(a) HR image

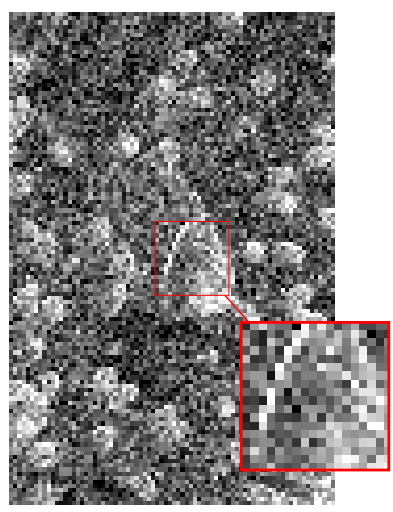

(b) One LR image

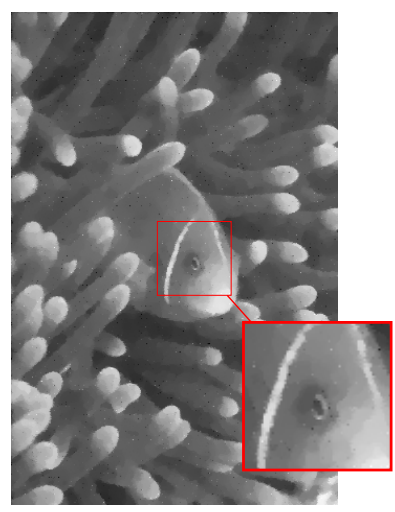

(c) Farsiu et al. [34]

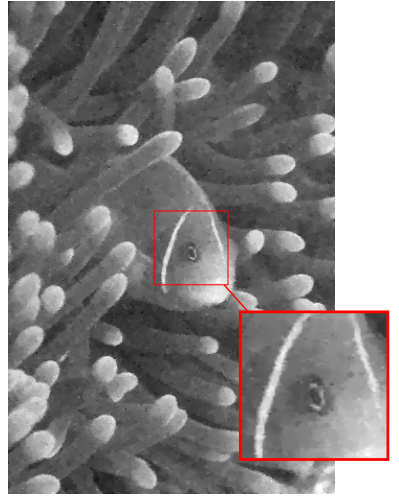

(d) Maiseli et al. [37]

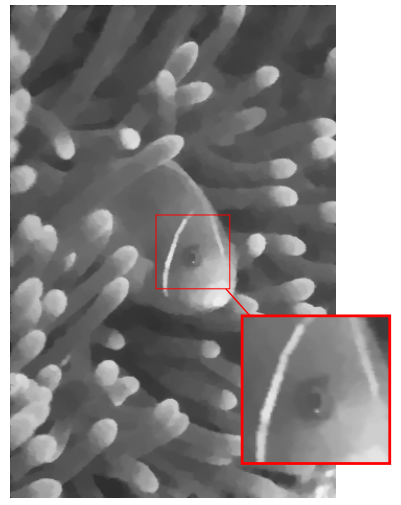

(e) Laghrib et al. [36]

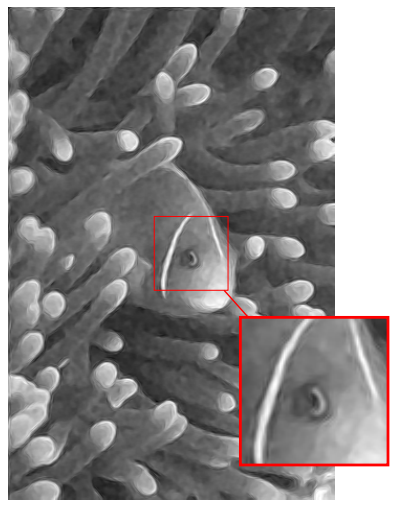

(f) our method with a first random choice of $k_{1}$, $k_{2}=40, \sigma=1.6$ and $\rho=3.2$ )

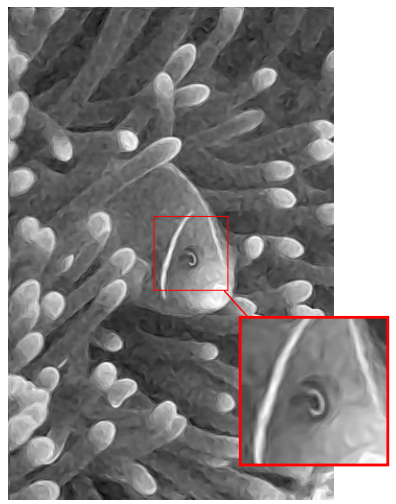

(g) our method with anther random choice of $k_{1}, k_{2}, \sigma$ and $\rho\left(k_{1}=24\right.$,
$k_{2}=10, \sigma=1.6$ and $\rho=3.2$ )

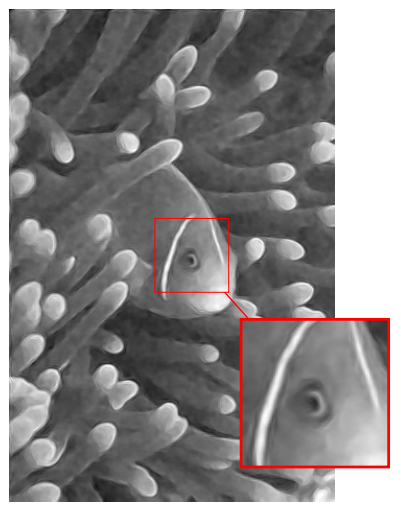

(h) our method with the optimal choice of $k_{1}, k_{2}$, $\sigma$ and $\rho$ such as $k_{1}=16$, $k_{2}=50, \sigma=1.6$ and $\rho=$ 3.2

Figure 10: The results obtained by applying 19 ifferent methods to LR (Fish sequence) with $\sigma=50$ noise. 


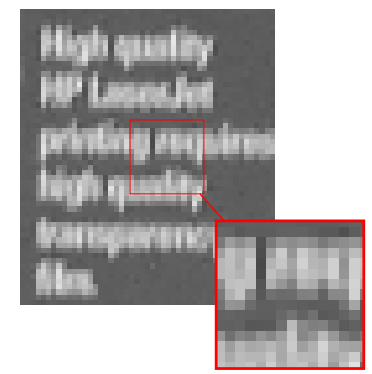

(a) One LR image

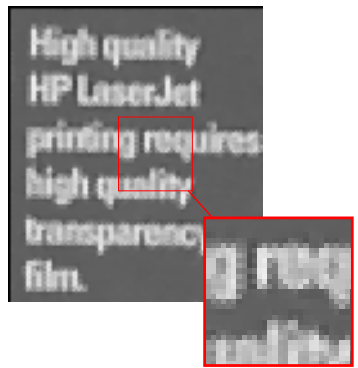

(b) Farsiu et al. [34]

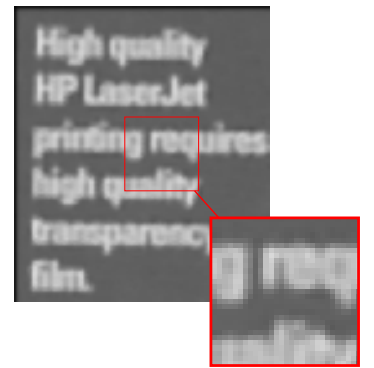

(c) Markina et al. [47]

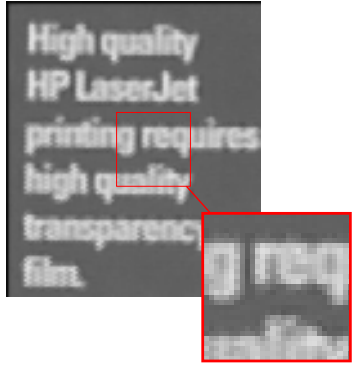

(d) Maiseli et al. [37]

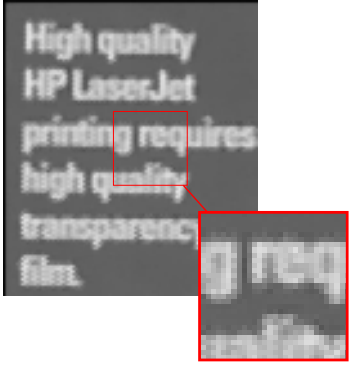

(e) our method

Figure 11: The results obtained by applying different methods to LR (Text sequence). 
Table 4: The PSNR table of the reconstructed Fish and Barbara images using different $\sigma$ noise values

\begin{tabular}{ccccc}
\hline Image & Method & $\sigma=10$ & $\sigma=30$ & $\sigma=50$ \\
\hline \hline \multirow{4}{*}{ Fish } & Farsiu [34] & 34.57 & 30.88 & 28.47 \\
& Maiseli et al. [37] & 34.79 & 30.98 & 28.74 \\
& Laghrib et. al [36] & 34.12 & 30.87 & 29.12 \\
& Our Method & $\mathbf{3 4 . 9 5}$ & $\mathbf{3 1 . 7 0}$ & $\mathbf{3 0 . 4 7}$ \\
\hline \multirow{4}{*}{ Barbara } & Farsiu [34] & 28.16 & 25.08 & 23.66 \\
& Maiseli et al. [37] & $\mathbf{2 9 . 1 3}$ & $\mathbf{2 5 . 8 6}$ & 24.03 \\
& Laghrib et. al [36] & 28.38 & 25.16 & 23.91 \\
& Our Method & 28.94 & 25.84 & $\mathbf{2 4 . 5 4}$ \\
\hline \hline
\end{tabular}

LR sequence. The new local method is based on a new tensor diffusion regularization with a linear isotropic behaviour in flat regions and a nonlinear filter near sharp edges. To approve this model, the existence and uniqueness of the proposed equation is proved. Simulated and real results demonstrate, visually, the performance of the new method and reveals the robustness with respect to blur and noise reduction compared with competitive methods. Moreover, we have shown that quantitatively the method generates always the best PSNR and SSIM values.

\section{Appendix}

Proof of theorem 2.1

1. Uniqueness:

We follow the same technique used in [38], let $X_{1}$ and $X_{2}$ be two solutions of the problem (6), for every $t \in[0, T]$ we have

$$
\begin{aligned}
& \frac{\partial X_{1}}{\partial t}(t, x)-\operatorname{div}\left(D\left(J_{\rho}\left(\nabla X_{1 \sigma}\right)\right) \nabla X_{1}\right)-\frac{1}{m} \sum_{k=1}^{m}\left(W F_{k} H\right)^{\top}\left(W F_{k} H X_{1}-X_{0}\right)=0 \\
& \frac{\partial X_{2}}{\partial t}(t, x)-\operatorname{div}\left(D\left(J_{\rho}\left(\nabla X_{2 \sigma}\right)\right) \nabla X_{2}\right)-\frac{1}{m} \sum_{k=1}^{m}\left(W F_{k} H\right)^{\top}\left(W F_{k} H X_{2}-X_{0}\right)=0
\end{aligned}
$$


let $w(t)=X_{1}(t)-X_{2}(t), D_{1}(t)=D\left(J_{\rho}\left(\nabla X_{1 \sigma}\right), D_{2}(t)=D\left(J_{\rho}\left(\nabla X_{2 \sigma}\right)\right.\right.$ and $N_{k}=\left(W F_{k} H\right)^{\top} W F_{k} H$, using equations (11) and (12), we obtain

$$
\frac{\partial w}{\partial t}(t)-\operatorname{div}\left(D_{1} \nabla w(t)\right)-\frac{1}{m} \sum_{k=1}^{m} N_{k} w(t)=\operatorname{div}\left(\left(D_{1}-D_{2}\right) \nabla X_{2}\right) .
$$

Then, multiplying the above inequality by $w(t)$, integrating over $\Omega$, and using the Green formula [50]

$$
\begin{gathered}
\frac{1}{2} \frac{\partial}{\partial t}\|w\|_{\mathbf{L}^{2}(\Omega)}^{2}+\int_{\Omega}\left\langle D_{1}(t) \nabla w(t), \nabla w(t)\right\rangle d x-\frac{1}{m} \sum_{k=1}^{m} \int_{\Omega}\left\langle N_{k} w(t) \cdot w(t)\right\rangle d x \\
=\int_{\Omega}\left\langle\left(D_{2}(t)-D_{1}(t)\right) \nabla X_{2}, \nabla w(t)\right\rangle d x
\end{gathered}
$$

Since the matrices $W, F_{k}$ and $H$ are symmetric positive-definite, there exists a positive constant $\eta_{k}$ such as

$$
\left\langle N_{k} w \cdot w\right\rangle \leq \eta_{k}|w|^{2}
$$

On the other hand, there exists a positive constant $\nu=\nu\left(\sigma,\left\|u_{0}\right\|_{\mathbf{L}^{\infty}(\Omega)}\right)$ (see [51] for more details), such that

$$
\nu|y|^{2} \leq\left\langle D_{1}(t) y, y\right\rangle \quad \forall y .
$$

From (15) and (16), the equation (14) becomes

$$
\begin{aligned}
& \frac{1}{2} \frac{\partial}{\partial t}\|w\|_{\mathbf{L}^{2}(\Omega)}^{2} \quad+\nu\|\nabla w(t)\|_{\mathbf{L}^{2}(\Omega)} \leq \frac{1}{m} \sum_{k=1}^{m} \int_{\Omega}\left\langle N_{k} w(t) \cdot w(t)\right\rangle d x \\
&+\left\|D_{1}(t)-D_{2}(t)\right\|_{\mathbf{L}^{\infty}(\Omega)}\left\|\nabla X_{2}(t)\right\|_{\mathbf{L}^{2}(\Omega)}\|\nabla w(t)\|_{\mathbf{L}^{2}(\Omega)} .
\end{aligned}
$$

Moreover, since the matrices $D_{1}(t)$ and $D_{2}(t)$ are smooth enough, we have

$$
\left\|D_{1}(t)-D_{2}(t)\right\|_{\mathbf{L}^{\infty}(\Omega)} \leq c\|w(t)\|_{\mathbf{L}^{2}(\Omega)} .
$$

Using the Young's inequality [52] in (17), we have

$$
\begin{gathered}
\frac{\partial}{\partial t}\|w\|_{\mathbf{L}^{2}(\Omega)}^{2}+2 \nu\|\nabla w(t)\|_{\mathbf{L}^{2}(\Omega)}^{2} \leq \frac{2}{m} \sum_{k=1}^{m} \eta_{k}\|w(t)\|_{\mathbf{L}^{2}(\Omega)} \\
+\frac{4 c^{2}}{\nu}\|w(t)\|_{\mathbf{L}^{2}(\Omega)}^{2}\left\|\nabla X_{2}(t)\right\|_{\mathbf{L}^{2}(\Omega)}^{2}+\nu\|\nabla w(t)\|_{\mathbf{L}^{2}(\Omega)}^{2},
\end{gathered}
$$


which is equivalent to

$$
\frac{\partial}{\partial t}\|w\|_{\mathbf{L}^{2}(\Omega)}^{2} \leq\left(\frac{2}{m} \sum_{k=1}^{m} \eta_{k}+\frac{4 c^{2}}{\nu}\left\|\nabla X_{2}(t)\right\|_{\mathbf{L}^{2}(\Omega)}^{2}\right)\|w(t)\|_{\mathbf{L}^{2}(\Omega)}^{2} .
$$

Using Gronwall's inequality [50], we deduce finally that

$$
\|w\|_{\mathbf{L}^{2}(\Omega)}^{2} \leq 0
$$

then $X_{1}=X_{2}$. Let's prove the existence of this solution.

1. Existence:

To prove the existence of a weak solution to the problem (6), we use the fixed point theorem of Schauder [53]. Firstly, we introduce the following functional space

$$
\mathbf{H}(0, T)=\left\{w \in \mathbf{L}^{2}\left((0, T) ; \mathbf{H}^{1}(\Omega)\right) ; \frac{\partial w}{\partial t} \in \mathbf{L}^{2}\left((0, T) ; \mathbf{H}^{1}(\Omega)^{\prime}\right)\right\},
$$

where $\mathbf{H}^{1}(\Omega)^{\prime}$ is the dual of the Sobolev space $\mathbf{H}^{1}(\Omega) . \mathbf{H}(0, T)$ is a Hilbert space equipped with the norm

$$
\|w\|_{\mathbf{H}(0, T)}=\|w\|_{\mathbf{L}^{2}\left((0, T) ; \mathbf{H}^{1}(\Omega)\right)}+\left\|\frac{\partial w}{\partial t}\right\|_{\mathbf{L}^{2}\left((0, T) ; \mathbf{H}^{1}(\Omega)^{\prime}\right)} .
$$

Let's $w \in \mathbf{H}(0, T) \cap \mathbf{L}^{\infty}\left((0, T) ; \mathbf{L}^{2}(\Omega)\right)$, such that

$$
\|w\|_{\mathbf{L}^{\infty}\left((0, T) ; \mathbf{L}^{2}(\Omega)\right)} \leq\left\|X_{0}\right\|_{\mathbf{L}^{2}(\Omega)},
$$

and let's define the variational problem associated to (6) with a fixed $w$

$$
\begin{gathered}
\left\langle\frac{\partial X}{\partial t}, v\right\rangle_{\mathbf{H}^{1}(\Omega)^{\prime}, \mathbf{H}^{1}(\Omega)}+\int_{\Omega}\left\langle D\left(J_{\rho}\left(\nabla w_{\sigma}\right)\right) \nabla X, \nabla Y\right\rangle d x= \\
\int_{\Omega} \frac{1}{m} \sum_{i=1}^{m} W^{\top} F_{k}^{\top} H^{\top}\left(W F_{k} H w-X_{0}\right) Y d x, \quad \forall Y \in \mathbf{H}^{1}(\Omega), \text { a.e in }[0, T](21)
\end{gathered}
$$

Therefore, based on the existence of parabolic equations results [45], we can prove that the problem (21) admits a unique solution $X_{w}$ in $\mathbf{H}(0, T)$, satisfying the following estimations

$$
\left\{\begin{array}{l}
\left\|X_{w}\right\|_{\mathbf{L}^{2}\left((0, T) ; \mathbf{H}^{1}(\Omega)\right)} \leq c_{1} \\
\left\|X_{w}\right\|_{\mathbf{L}^{\infty}\left((0, T) ; \mathbf{L}^{2}(\Omega)\right)} \leq\left\|X_{0}\right\|_{\mathbf{L}^{2}(\Omega)}, \\
\left\|\frac{\partial X_{w}}{\partial t}\right\|_{\mathbf{L}^{2}\left((0, T) ; \mathbf{H}^{1}(\Omega)^{\prime}\right)} \leq c_{2}
\end{array}\right.
$$


where the constants $c_{1}$ and $c_{2}$ depends on $\rho, \sigma, W, F, H$ and $X_{0}$. These estimations lead to define the subset $\mathbf{H}_{0}$ of $\mathbf{H}(0, T)$ as

$$
\begin{gathered}
\mathbf{H}_{0}=\left\{w \in \mathbf{H}(0, T), w(0)=X_{0} ;\|w\|_{\mathbf{L}^{2}\left((0, T) ; \mathbf{H}^{1}(\Omega)\right)} \leq c_{1},\right. \\
\left.\|w\|_{\mathbf{L}^{\infty}\left((0, T) ; \mathbf{L}^{2}(\Omega)\right)} \leq\left\|X_{0}\right\|_{\mathbf{L}^{2}(\Omega)},\left\|\frac{\partial w}{\partial t}\right\|_{\mathbf{L}^{2}\left((0, T) ; \mathbf{H}^{1}(\Omega)^{\prime}\right)} \leq c_{2}\right\}
\end{gathered}
$$

Let $U: w \rightarrow U(w)=X_{w}$ be a mapping from $\mathbf{H}_{0}$ to $\mathbf{H}_{0}$. It's clear that $\mathbf{H}_{0}$ is a non empty, convex, and weakly compact subspace of $\mathbf{H}(0, T)$ [52]. In order to use the classical Schauder's fixed-point theorem, we need first to prove that the mapping $w \rightarrow U(w)$ is weakly continuous. Indeed, let $\left(w_{n}\right)_{n}$ be a sequence that converges weakly to $w$ in $\mathbf{H}_{0}$ and $X_{n}=U\left(w_{n}\right)$. Using the compact inclusions of Sobolev space [50] and by the estimations (22), there exists a subsequence noted also $\left(w_{n}\right)$ and $\left(X_{n}\right)$ such that

$$
\begin{aligned}
\frac{\partial w_{n}}{\partial t} & \rightarrow \frac{\partial w}{\partial t} \quad \text { in } \mathbf{L}^{2}\left((0, T) ; \mathbf{H}^{1}(\Omega)^{\prime}\right), \\
X_{n} & \rightarrow X \quad \text { in } \mathbf{L}^{2}\left((0, T) ; \mathbf{L}^{2}(\Omega)\right), \\
\nabla X_{n} & \rightarrow \nabla X \quad \text { in }\left(\mathbf{L}^{2}\left((0, T) ; \mathbf{L}^{2}(\Omega)\right)\right)^{2}, \\
w_{n} & \rightarrow w \quad \text { in } \mathbf{L}^{2}\left((0, T) ; \mathbf{L}^{2}(\Omega)\right), \\
D\left(J_{\rho}\left(\nabla w_{n \sigma}\right)\right) & \rightarrow D\left(J_{\rho}\left(\nabla w_{\sigma}\right)\right) \quad \text { in } \mathbf{L}^{2}\left((0, T) ; \mathbf{L}^{2}(\Omega)\right), \\
& X_{n}(0) \rightarrow X(0) \quad \text { in } \mathbf{H}^{1}(\Omega)^{\prime}
\end{aligned}
$$

Using the limit as $n \rightarrow \infty$, we obtain $X=X_{w}=U(w)$. Moreover, by the uniqueness of the solution of (6), the sequence $X_{n}=U\left(w_{n}\right)$ converge weakly to $X=U(w)$, this prove the continuity of $U$. Finally, from the Schauder's fixed-point theorem, there exists a function $w \in \mathbf{H}_{0}$ such that $w=S(w)=$ $X_{w}$. Moreover, since $X \in \mathbf{L}^{2}\left((0, T) ; \mathbf{H}^{1}(\Omega)\right)$ and $\frac{\partial X}{\partial t} \in \mathbf{L}^{2}\left((0, T) ; \mathbf{H}^{1}(\Omega)^{\prime}\right)$, by the Aubin's theorem [54], we can deduce that $X \in C\left((0, T) ; \mathbf{L}^{2}(\Omega)\right)$.

\section{Acknowledgements}

We are grateful to the anonymous referee for the corrections and useful suggestions that have improved this article. 


\section{Compliance with Ethical Standards}

- Funding: This research was entirely funded by the respective institutions of the authors.

- Conflict of interest: The authors declare that they have no conflict of interest.

- Neither human participants nor animals are involved in this research.

\section{Bibliography}

[1] Super-Resolution Imaging, Digital Imaging and Computer Vision, CRC Press, 2010.

[2] A locally adaptive 11- 12 norm for multi-frame super-resolution of images with mixed noise and outliers, Signal Processing 105 (2014) 156 - 174.

[3] D. Capel, A. Zisserman, Computer vision applied to super-resolution, IEEE Signal Process. Mag 20 (2003) 75-86.

[4] M. Protter, M. Elad, H. Takeda, P. Milanfar, Generalizing the nonlocalmeans to super-resolution reconstruction, IEEE Transactions on Image Processing 18 (1) (2009) 36-51.

[5] R. M. Bahy, G. I. Salama, T. A. Mahmoud, Adaptive regularizationbased super resolution reconstruction technique for multi-focus lowresolution images, Signal Processing 103 (2014) 155-167.

[6] X. Li, Y. Hu, X. Gao, D. Tao, B. Ning, A multi-frame image superresolution method, Signal Processing 90 (2) (2010) 405-414.

[7] A. Laghrib, A. Hakim, S. Raghay, M. EL Rhabi, Robust super resolution of images with non-parametric deformations using an elastic registration, Applied Mathematical Sciences 8 (179) (2014) 8897-8907.

[8] E. Sardis, A. Voulodimos, V. Anagnostopoulos, C. Lalos, A. Doulamis, D. Kosmopoulos, An industrial video surveillance system for quality assurance of a manufactory assembly, in: Proceedings of the 3rd International Conference on PErvasive Technologies Related to Assistive Environments, ACM, 2010, p. 66. 
[9] D. I. Kosmopoulos, N. D. Doulamis, A. S. Voulodimos, Bayesian filter based behavior recognition in workflows allowing for user feedback, Computer Vision and Image Understanding 116 (3) (2012) 422-434.

[10] K. Makantasis, K. Karantzalos, A. Doulamis, N. Doulamis, Deep supervised learning for hyperspectral data classification through convolutional neural networks, in: 2015 IEEE International Geoscience and Remote Sensing Symposium (IGARSS), IEEE, 2015, pp. 4959-4962.

[11] A. J. Tatem, H. G. Lewis, P. M. Atkinson, M. S. Nixon, Super-resolution land cover pattern prediction using a hopfield neural network, Remote Sensing of Environment 79 (1) (2002) 1-14.

[12] L. Zhang, H. Zhang, H. Shen, P. Li, A super-resolution reconstruction algorithm for surveillance images, Signal Processing 90 (3) (2010) 848859 .

[13] F. C. Lin, C. B. Fookes, V. Chandran, S. Sridharan, Investigation into optical flow super-resolution for surveillance applications.

[14] F. Stanco, S. Battiato, G. Gallo, Digital imaging for cultural heritage preservation: Analysis, restoration, and reconstruction of ancient artworks, CRC Press, 2011.

[15] A. Doulamis, N. Doulamis, C. Ioannidis, C. Chrysouli, N. Grammalidis, K. Dimitropoulos, C. Potsiou, E. K. Stathopoulou, M. Ioannides, 5d modelling: An efficient approach for creating spatiotemporal predictive 3d maps of large-scale cultural resources, ISPRS Annals of the Photogrammetry, Remote Sensing and Spatial Information Sciences 2 (5) (2015) 61.

[16] J. Yang, T. Huang, Image super-resolution: Historical overview and future challenges, Super-resolution imaging (2010) 20-34.

[17] A. J. Tatem, H. G. Lewis, P. M. Atkinson, M. S. Nixon, Super-resolution target identification from remotely sensed images using a hopfield neural network, IEEE Transactions on Geoscience and Remote Sensing 39 (4) (2001) 781-796. 
[18] S. C. Park, M. K. Park, M. G. Kang, Super-resolution image reconstruction: a technical overview, IEEE Signal Processing Magazine 20 (3) (2003) 21-36.

[19] R. Y. Tsai, T. S. Huang, Multiframe image restoration and registration, In: Advances in Computer Vision and Image Processing, ed. T.S.Huang. Greenwich, CT, JAI Press.

[20] S. Borman, R. L. Stevenson, Super-resolution from image sequences-a review, in: mwscas, IEEE, 1998, p. 374.

[21] M. Elad, Y. Hel-Or, A fast super-resolution reconstruction algorithm for pure translational motion and common space-invariant blur, IEEE Transactions on Image Processing 10 (8) (2001) 1187-1193.

[22] N. Nguyen, P. Milanfar, G. Golub, A computationally efficient superresolution image reconstruction algorithm, IEEE Transactions on Image Processing 10 (4) (2001) 573-583.

[23] S. Villena, M. Vega, R. Molina, A. K. Katsaggelos, Bayesian superresolution image reconstruction using an 11 prior, in: Image and Signal Processing and Analysis, 2009. ISPA 2009. Proceedings of 6th International Symposium on, IEEE, 2009, pp. 152-157.

[24] S. D. Babacan, R. Molina, A. K. Katsaggelos, Variational bayesian super resolution, IEEE Transactions on Image Processing 20 (4) (2011) 984999.

[25] O. A. Omer, T. Tanaka, Region-based weighted-norm with adaptive regularization for resolution enhancement, Digital Signal Processing 21 (4) (2011) 508-516.

[26] S. Zhao, H. Liang, M. Sarem, A generalized detail-preserving superresolution method, Signal Processing 120 (2016) 156-173.

[27] S. D. Babacan, R. Molina, A. K. Katsaggelos, Parameter estimation in tv image restoration using variational distribution approximation, IEEE Transactions on Image Processing 17 (3) (2008) 326-339.

[28] A. Panagiotopoulou, V. Anastassopoulos, Regularized super-resolution image reconstruction employing robust error norms, Optical Engineering 48 (11) (2009) 117004-117004. 
[29] V. Patanavijit, S. Jitapunkul, A robust iterative multiframe superresolution reconstruction using a huber bayesian approach with hubertikhonov regularization, in: Intelligent Signal Processing and Communications, 2006. ISPACS'06. International Symposium on, IEEE, 2006, pp. $13-16$.

[30] N. A. El-Yamany, P. E. Papamichalis, Robust color image superresolution: An adaptive m-estimation framework, Journal on Image and Video Processing 2008 (2008) 16.

[31] T. Q. Pham, L. v Vliet, K. Schutte, Robust super-resolution by minimizing a gaussian-weighted 12 error norm, in: Journal of Physics: Conference Series, Vol. 124, IOP Publishing, 2008, p. 012037.

[32] S. Tourbier, X. Bresson, P. Hagmann, J.-P. Thiran, R. Meuli, M. B. Cuadra, An efficient total variation algorithm for super-resolution in fetal brain mri with adaptive regularization, NeuroImage 118 (2015) $584-597$.

[33] V. Patanavijit, S. Jitapunkul, A lorentzian stochastic estimation for a robust iterative multiframe super-resolution reconstruction with lorentzian-tikhonov regularization, EURASIP Journal on Advances in Signal Processing 2007 (1) (2007) 1-21.

[34] S. Farsiu, D. Robinson, M. Elad, P. Milanfar, Advances and challenges in super-resolution, International Journal of Imaging Systems and Technology 14 (2) (2004) 47-57.

[35] X. Zeng, L. Yang, A robust multiframe super-resolution algorithm based on half-quadratic estimation with modified btv regularization, Digital Signal Processing 23 (1) (2013) 98-109.

[36] A. Laghrib, A. Hakim, S. Raghay, A combined total variation and bilateral filter approach for image robust super resolution, EURASIP Journal on Image and Video Processing 2015 (1) (2015) 1-10.

[37] B. J. Maiseli, N. Ally, H. Gao, A noise-suppressing and edge-preserving multiframe super-resolution image reconstruction method, Signal Processing: Image Communication 34 (2015) 1-13. 
[38] J. Weickert, Anisotropic diffusion in image processing, Vol. 1, Teubner Stuttgart, 1998.

[39] J. Weickert, B. T. H. Romeny, M. Viergever, et al., Efficient and reliable schemes for nonlinear diffusion filtering, IEEE Transactions on Image Processing 7 (3) (1998) 398-410.

[40] J. Weickert, C. Schnörr, A theoretical framework for convex regularizers in pde-based computation of image motion, International Journal of Computer Vision 45 (3) (2001) 245-264.

[41] P. Perona, J. Malik, Scale-space and edge detection using anisotropic diffusion, IEEE Transactions on Pattern Analysis and Machine Intelligence 12 (7) (1990) 629-639.

[42] L. I. Rudin, S. Osher, E. Fatemi, Nonlinear total variation based noise removal algorithms, Physica D: Nonlinear Phenomena 60 (1) (1992) 259268.

[43] Y.-L. You, W. Xu, A. Tannenbaum, M. Kaveh, Behavioral analysis of anisotropic diffusion in image processing, IEEE Transactions on Image Processing 5 (11) (1996) 1539-1553.

[44] J. Weickert, Coherence-enhancing diffusion of colour images, Image and Vision Computing 17 (3) (1999) 201-212.

[45] G. Aubert, P. Kornprobst, Mathematical problems in image processing: partial differential equations and the calculus of variations, Vol. 147, Springer Science \& Business Media, 2006.

[46] M. Werlberger, W. Trobin, T. Pock, A. Wedel, D. Cremers, H. Bischof, Anisotropic huber-11 optical flow., in: BMVC, Vol. 1, 2009, p. 3.

[47] A. Marquina, S. J. Osher, Image super-resolution by tv-regularization and bregman iteration, Journal of Scientific Computing 37 (3) (2008) $367-382$.

[48] Z. Wang, A. C. Bovik, Mean squared error: love it or leave it? a new look at signal fidelity measures, IEEE Signal Processing Magazine 26 (1) (2009) 98-117. 
[49] Z. Wang, A. C. Bovik, H. R. Sheikh, E. P. Simoncelli, Image quality assessment: from error visibility to structural similarity, IEEE Transactions on Image Processing 13 (4) (2004) 600-612.

[50] H. Attouch, G. Buttazzo, G. Michaille, Variational analysis in Sobolev and BV spaces: applications to PDEs and optimization, Vol. 17, Siam, 2014.

[51] J. Weickert, Scale-space properties of nonlinear diffusion filtering with a diffusion tensor, Citeseer, 1994.

[52] H. Brezis, Functional analysis, Sobolev spaces and partial differential equations, Springer Science \& Business Media, 2010.

[53] J. T. Schwartz, Nonlinear functional analysis, CRC Press, 1969.

[54] J.-P. Aubin, Un théoreme de compacité, CR Acad. Sci. Paris 256 (24) (1963) 5042-5044. 